\title{
Morphological and molecular variations induce mitochondrial dysfunction as a possible underlying mechanism of athletic amenorrhea
}

\author{
RUO-HONG XIONG $^{1^{*}}$, SHI-LEI WEN ${ }^{2 *}$, QIANG WANG ${ }^{1}$, HONG-YING ZHOU $^{2}$ and SHI FENG ${ }^{2}$ \\ ${ }^{1}$ Chengdu Sport Institute; ${ }^{2}$ Department of Human Anatomy, West China School of \\ Basic Medical and Forensic Medicine, Sichuan University, Chengdu, Sichuan 610041, P.R. China
}

Received March 27, 2017; Accepted September 5, 2017

DOI: $10.3892 /$ etm.2017.5469

\begin{abstract}
Female athletes may experience difficulties in achieving pregnancy due to athletic amenorrhea (AA); however, the underlying mechanisms of AA remain unknown. The present study focuses on the mitochondrial alteration and its function in detecting the possible mechanism of AA. An AA rat model was established by excessive swimming. Hematoxylin and eosin staining, and transmission electron microscopic methods were performed to evaluate the morphological changes of the ovary, immunohistochemical examinations and radioimmunoassays were used to detect the reproductive hormones and corresponding receptors. Reverse transcription-quantitative polymerase chain reaction (RT-qPCR) was used to test the mtDNA copy number. PCR and western blot analysis were used to test the expression of ND2. The change of morphological features of the rat ovaries revealed evident abnormalities. Particularly, the features of the mitochondria were markedly altered. In addition, reproductive hormones in the serum and tissues of AA rats were also detected to evaluate the function of the ovaries, and the levels of these hormones were significantly decreased. Furthermore, the mitochondrial DNA copy number (mtDNA) and expression of NADH dehydrogenase subunit 2 (ND2) were quantitated by qPCR or western blot analysis. Accordingly, the mtDNA copy number and expression of ND2 expression were markedly reduced in the AA rats. In conclusion, mitochondrial dysfunction in AA may affect the cellular energy supply and,
\end{abstract}

Correspondence to: Professor Shi Feng or Professor Hong-Ying Zhou, Department of Human Anatomy, West China School of Basic Medical and Forensic Medicine, Sichuan University, 17 South Renmin Road, Chengdu, Sichuan 610041, P.R. China

E-mail: jetmork@126.com

E-mail: eaglezhyxzy@163.com

*Contributed equally

Key words: athletic menstrual cycle irregularity, mitochondrial dysfunction, mtDNA copy number therefore, result in dysfunction of the ovary. Thus, mitochondrial dysfunction may be considered as a possible underlying mechanism for the occurrence of AA.

\section{Introduction}

An increasing number of athletes are suffering from sports-associated diseases in the competitive sports. For professional female athletes, the incidence of athletic amenorrhea (AA) is particularly high, at a rate of $65-69 \%$ in athletes such as dancers and distance runners compared with $2-5 \%$ in normal women (1). The high AA in female athletes suggests that achieving pregnancy may be challenging. The current treatment methods primarily including increasing weight and decreasing exercise, replenishing vitamins and minerals, and reinforcing strength training (2). The normal menstrual cycle is regulated by the hypothalamic-pituitary-ovarian axis, and the ovary is one of the most important organs in this axis (3). Thus, it is important to investigate the underlying mechanism of ovary dysfunction in AA.

The mitochondrion is the organelle of energy supply in eukaryotic organisms. Mitochondrial abnormity or dysfunction has been reported to be involved in various diseases (4). To some of these diseases, the dysfunction of mitochondrion is considered as a key mechanism to cause the dysfunction of the organ, thus it was conjectured that the dysfunction of mitochondrion may cause the dysfunction of ovary, which may result in AA. The number of mitochondria and the mitochondrial DNA (mtDNA) copy number may vary among different types of cells and tissues. Furthermore, the mitochondria and mtDNA copy number may be altered during cell differentiation and aging. It has also been reported in previous studies that the alteration of mtDNA participates in the regulation of ovarian function (5-8). Therefore, it is likely that the dysfunction of the mitochondrion may be associated AA.

Thus, the present study aimed to describe the alterations of the mitochondria in the ovaries of AA rats, morphologically and at the molecular level. In addition, the study attempted to investigate the possible mechanism underlying ovary dysfunction by determining the mitochondrial variations. 


\section{Materials and methods}

Animals and experimental design. A total of 40 healthy female Sprague Dawley rats (3-month-old, weight, $250 \pm 20 \mathrm{~g}$ ) with a normal menstrual cycle were obtained from the West China Medical Experimental Animal Center of Sichuan University (Chengdu, China). All animals used in the present study were kept under a 12-h light/dark cycles at a constant temperature and humidity $\left(25 \pm 2^{\circ} \mathrm{C}, 60-75 \%\right)$ with free access to chow and water. The animal procedures were approved by the Animal Use and Care Committee of Sichuan University and conducted according to the regulations.

The animals were randomly divided into the control and AA groups ( $\mathrm{n}=20$ in each group). Rats in the control group were maintained under a 12-h light/dark cycles at a constant temperature and humidity $\left(25 \pm 2^{\circ} \mathrm{C}, 60-75 \%\right)$, without swimming training, while rats in the AA group were subjected to excessive swimming for $2 \mathrm{~h}$ per day for 6 days per week (with 1-day break) for 3 weeks. Vaginal exfoliative cystoscopy was conducted at 8:00-9:00 a.m. each day for observation of any possible alterations in the menstrual cycle.

Specimen collection. After excessive swimming training for 3 weeks, the rats were sacrificed and the ovary tissues were collected. Half of the ovary was fixed in $4 \%$ neutral buffered paraformaldehyde at $4^{\circ} \mathrm{C}$ for at least $24 \mathrm{~h}$ for histopathological and immunohistochemical examinations. The samples were cut into $5-\mu \mathrm{m}$-thick sections. Hematoxylin and eosin staining was performed for histopathological exam at $25^{\circ} \mathrm{C}$ and slides were observed under the microscope. Images were captured at a magnification of $x 100$. The remaining part of the ovary was immediately frozen in liquid nitrogen and stored at $-80^{\circ} \mathrm{C}$ for mRNA and protein expression analysis. Furthermore, serum samples were also collected and stored at $-80^{\circ} \mathrm{C}$ for reproductive hormones analysis. The ratio (ovary weight/body weight $\mathrm{x} 100 \%$ ) was also calculated for each rat. Samples for TEM were fixed in $2.5 \%$ glutaraldehyde at $4^{\circ} \mathrm{C}$ for at least $12 \mathrm{~h}$. Sections $(0.05-\mu \mathrm{m}$-thick) were stained with $0.5 \%$ lead citrate for $20-30 \mathrm{~min}$ at $20^{\circ} \mathrm{C}$. Images were captured at a magnification of $x 12,000$.

Detection of reproductive hormones in the serum by radioimmunoassay. The concentration of reproductive hormones in the serum, including estradiol $\left(\mathrm{E}_{2}\right)$, progesterone (P), follicle-stimulating hormone (FSH), luteotropic hormone $(\mathrm{LH})$ and testosterone $(\mathrm{T})$, was measured and quantified using radioimmunoassay kits (Jingmei Life Sciences Inc., Beijing, China), according to the manufacturer's protocol.

Determination of reproduction-associated factors in ovarian sections by immunohistochemical assay. The deparaffinated ovarian tissue sections (5- $\mu \mathrm{m}$-thick) were incubated with $3 \%$ $\mathrm{H}_{2} \mathrm{O}_{2}$ at $37^{\circ} \mathrm{C}$ for $10 \mathrm{~min}$ to quench the endogenous peroxidase. Subsequent to blocking by $10 \%$ goat serum at room temperature for $20 \mathrm{~min}$, the sections were incubated with primary antibodies, including $17-\mathrm{b}$ estradiol $\left(\mathrm{E}_{2}\right.$; dilution: 1:300; cat. no. sc-69958; Santa Cruz Biotechnology, Inc., Dallas, TX, USA), estrogen receptor (ER; dilution: 1:250; cat. no. A000786; Boster Biological Technology, Ltd., Wuhan, China), progesterone receptor (PR; dilution: 1:300; cat. no. PB0077; Boster Biological Technology, Ltd.) overnight at $4^{\circ} \mathrm{C}$, followed by incubation with horseradish-peroxidase (HRP)-conjugated secondary antibodies (cat. nos. SP-9001 or SP-9002; OriGene Technologies, Inc., Beijing, China) at $37^{\circ} \mathrm{C}$ for $30 \mathrm{~min}$. Subsequently, the signals were detected using a diaminobenzidine substrate kit (cat. nos. SP-9001 or SP-9002; OriGene Technologies, Inc.). Positive staining in the images was indicated by brown staining in the cytoplasm or nucleus. Five fields-of-view were randomly selected for assessment in each tissue section. The images were observed and captured using a white-light microscope at a magnification of $\mathrm{x} 400$.

Analysis of mtDNA copy number and NADH dehydrogenase subunit 2 (ND2) mRNA expression in rat ovarian tissues. The mtDNA copy number was measured by qPCR using a SYBR $^{\mathrm{TM}}$ Green PCR Master Mix (cat. no. 4309155; Applied Biosystems; Thermo Fisher Scientific, Inc., Waltham, MA, USA). Briefly, the DNA specimens were prepared using the TIANamp Genomic DNA kit (cat. no. DP304; Tiangen Biotech Co., Ltd., Beijing, China). The mtDNA copy number was assessed by amplification of the mitochondrial D-loop against the reference gene, GAPDH. The primers used were as follows: D-loop forward, 5'-GGTTCTTACTTCAGGGCC ATCA-3' and reverse 5'-GATTAGACCCGTTACCATCGA GAT-3'; GAPDH, forward 5'-CGGGAAATCGTGCGTGAC AT-3' and reverse 5'-GAAGGAAGGCTGGAAGAGTG-3'. The following PCR cycling conditions were performed: An activation step at $95^{\circ} \mathrm{C}$ for $3 \mathrm{~min}$, followed by 35 cycles of $25 \mathrm{sec}$ at $95^{\circ} \mathrm{C}, 25 \mathrm{sec}$ at $56^{\circ} \mathrm{C}$ and $30 \mathrm{sec}$ at $72^{\circ} \mathrm{C}$.

For ND2 mRNA detection, total RNA was extracted with TRIzol $^{\mathrm{TM}}$ Reagent (cat. no. 15596018; Thermo Fisher Scientific, Inc.), and the concentration and quality of total RNA was measured with a spectrophotometer. First strand cDNA was obtained using a RevertAid First Strand cDNA Synthesis Kit (cat. no. K1622; Thermo Fisher Scientific, Inc.). The mRNA expression levels of ND2 were detected by qPCR using the following primers: ND2, forward 5'-TTATCCTCT TATCCGTCCTC-3' and reverse 5'-TGTTAAGTCGAAGGG AGC-3'. The expression levels of genes were calculated using the $2^{-\Delta \Delta \mathrm{Cq}}$ method (6). The relative ratio of the D-loop and GAPDH expression was used as the relative abundance of the mitochondrial genome.

Quantification of ND2 protein expression in rat ovarian tissues by western blot analysis. Frozen ovarian tissues were homogenized and whole proteins were extracted by ice-cold radioimmunoprecipitation assay buffer (Beyotime Institute of Biotechnology, Nanjing, China). A BCA kit was used to quantify the protein concentration. Equal amounts of protein $(50 \mu \mathrm{g})$ from each sample were resolved by $12 \%$ SDS-PAGE, transferred to polyvinylidene difluoride membranes (EMD Millipore, Billerica, MA, USA) and blocked with 5\% non-fat powdered milk in Tris-buffered saline/Tween 20 (containing $20 \mathrm{mM}$ Tris- $\mathrm{HCl}, \mathrm{pH} 7.5,150 \mathrm{mM} \mathrm{NaCl}$ and $0.1 \%$ Tween-20). The membranes were then incubated with goat anti-ND2 polyclonal antibody (dilution: 1:500; cat. no. sc-20496; Santa Cruz Biotechnology, Inc., Dallas, TX, USA) for $2 \mathrm{~h}$ at room temperature, followed by incubation with an HRP-conjugated rabbit anti-goat IgG secondary antibody (dilution: 1:10,000; cat. no. SA00001-4; ProteinTech Group, Inc., Wuhan, China) 
for $1.5 \mathrm{~h}$ at room temperature. For GAPDH detection, membranes were incubated with HRP-conjugated GAPDH antibody (dilution: 1:20,000; cat. no. HRP-60004; ProteinTech Group, Inc.) for $2 \mathrm{~h}$ at room temperature. The membranes were then visualized using an enhanced chemiluminescence detection kit (Beyotime Institute of Biotechnology). The protein expression was normalized to that of GAPDH, which was used as an internal control.

Statistical analysis. All data are expressed as the mean \pm standard deviation, and statistical calculations were performed with the statistical package SPSS version 18.0 (SPSS, Inc., Chicago, IL, USA). For comparisons between the two groups, Student's t-test was applied. The presence of a statistically significant difference was indicated by $\mathrm{P}<0.05$.

\section{Results}

Morphological variations to ovaries in the AA group. The gross morphological features of the rat ovaries were examined by hematoxylin and eosin staining and transmission electron microscopy (TEM) following sacrifice at week 3 (Fig. 1A). Several ovarian follicles and corpus luteum structures were identified in the ovaries of the control group. By contrast, evidently reduced ovarian follicles and corpus luteum structures were observed in the AA group. These observations were also confirmed by hematoxylin and eosin staining. Furthermore, the ultrastructure of the mitochondria was also observed. In the AA group, the mitochondria presented marked abnormal alterations, including swelling of the mitochondrial membrane, disappearance of the mitochondrial cristae and decrease of the mitochondrial number, in comparison with the control group. However, no evident changes in other structures were detected between the two groups (Fig. 1A). Notably, the ovary weight/body weight ratio of the AA group was significantly decreased when compared with that in the control group $(0.0350 \pm 0.0127 \%$ vs. $0.0507 \pm 0.0105 \%$, respectively; $\mathrm{P}<0.01$; Fig. 1B).

Decrease in the reproductive hormone levels in the ovaries of $A$ A rats. Immunohistochemical assay was applied to detect the expression of reproductive hormones in the ovarian tissues, including $\mathrm{E}_{2}$, ER and PR (Fig. 2A). The expression levels of these factors were calculated in both the granular and luteal cells of the ovaries. Compared with the control group, the optical density in the immunohistochemical images of these hormones was significantly decreased in the AA group in the two cell types $(\mathrm{P}<0.05$ or $\mathrm{P}<0.01$; Fig. $2 \mathrm{~B})$.

Reduction of serum reproductive hormone levels in AA rats. Radioimmunoassay was performed in order to detect the expression levels of five reproductive hormones in the rat serum, including $\mathrm{E}_{2}, \mathrm{P}, \mathrm{FSH}, \mathrm{LH}$ and $\mathrm{T}$. The levels of these hormones were significantly decreased in the AA group when compared with the control group $(\mathrm{P}<0.05$; Table I). These results indicate depressed ovarian function and reproductive capability in the AA rats.

Decrease of relative mtDNA copy number in AA ovarian tissues. The mtDNA copy number of ovarian tissues was
A
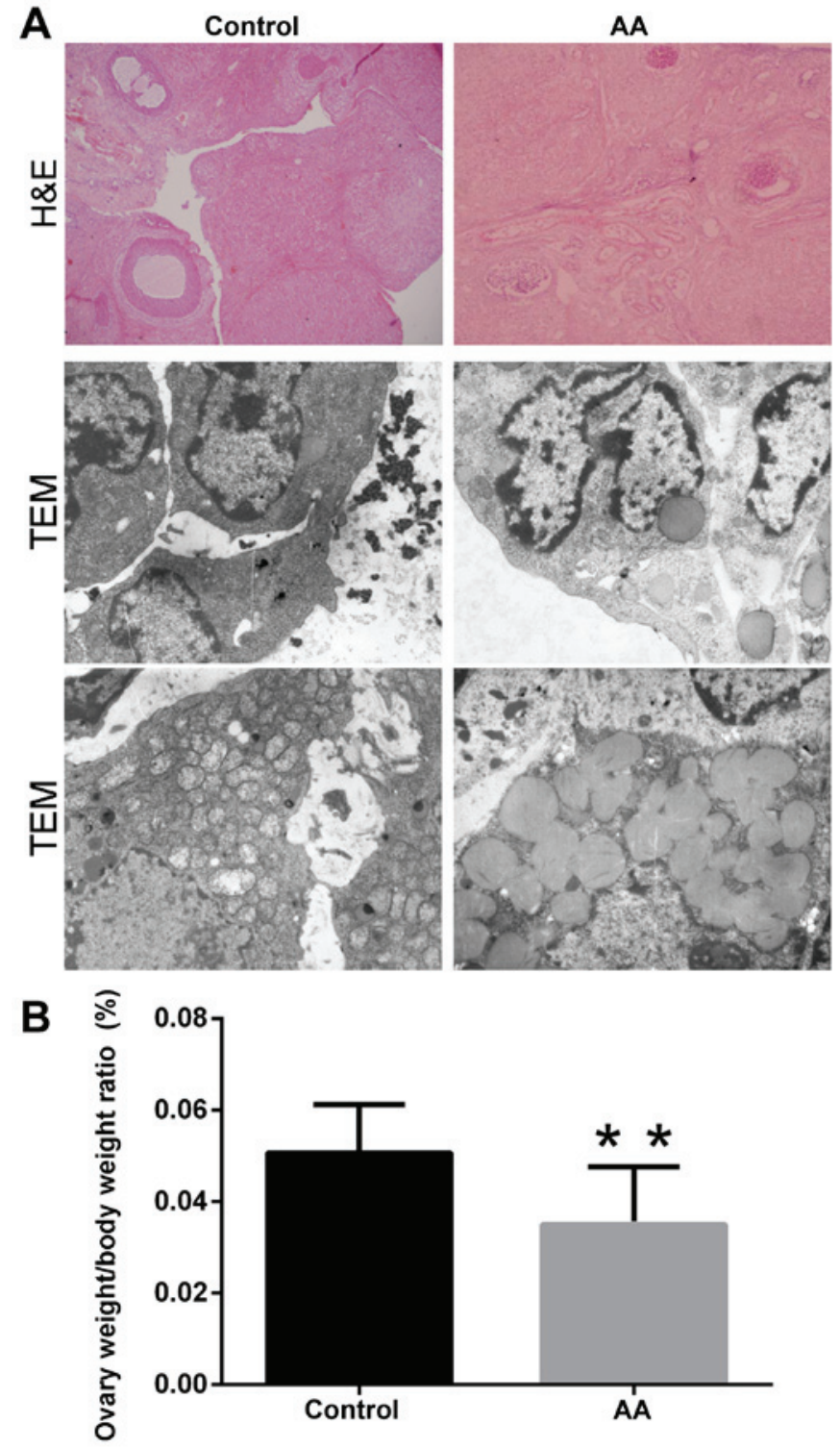

Figure 1. Morphological variations of the ovaries of AA rats. (A) H\&E staining and TEM images of rat ovaries. (B) Decreased ovary coefficient in the AA group. $\mathrm{n}=20$ in each group. ${ }^{* *} \mathrm{P}<0.01$ vs. control group. $\mathrm{H} \& \mathrm{E}$, hematoxylin and eosin; TEM, transmission electron microscopy; AA, athletic amenorrhea.

quantified in the AA and control rats. Following statistical analysis, the results revealed that the relative mtDNA copy numbers of ovarian tissues were significantly decreased in the AA rats compared with the control rats $(57.65 \pm 19.20$ vs. 102.42 \pm 24.92 , respectively; Fig. $3 \mathrm{~A}$ and $\mathrm{B}$; $\mathrm{P}<0.01)$. The results indicated that the function level of the mitochondria was higher in control group than that in AA group.

Reduction of ND2 expression in AA rat ovarian tissues. The expression of ND2 was analyzed by western blot analysis to evaluate the activity of mitochondria in the ovarian tissues of AA rats. Following statistical analysis, the results revealed that the relative protein levels of ND2 in the AA group were markedly decreased when compared with those in the control group. (Fig. 3C and D; $\mathrm{P}<0.01$ ). These findings suggested that the oxidative phosphorylase activity was higher in the control 
Table I. Reproductive hormone levels in the rat serum.

\begin{tabular}{lcccccc}
\hline Group & $\mathrm{n}$ & $\mathrm{E}_{2}(\mathrm{ng} / \mathrm{ml})$ & $\mathrm{P}(\mathrm{pg} / \mathrm{ml})$ & FSH $(\mathrm{mIU} / \mathrm{ml})$ & $\mathrm{LH}(\mathrm{mIU} / \mathrm{ml})$ & $\mathrm{T}(\mathrm{ng} / \mathrm{dl})$ \\
\hline Control & 20 & $1.56 \pm 0.48$ & $73.81 \pm 23.42$ & $8.57 \pm 1.22$ & $2.13 \pm 0.30$ & $19.41 \pm 4.51$ \\
AA & 20 & $1.27 \pm 0.34^{\mathrm{a}}$ & $45.70 \pm 18.44^{\mathrm{a}}$ & $7.34 \pm 0.99^{\mathrm{a}}$ & $1.32 \pm 0.24^{\mathrm{a}}$ & $15.60 \pm 3.07^{\mathrm{a}}$ \\
\hline
\end{tabular}

${ }^{\mathrm{a}} \mathrm{P}<0.05$ vs. the control group. Data are reported as the mean \pm standard deviation. E2, estradiol; P, progesterone; FSH, follicle-stimulating hormone; LH, luteotropic hormone; T, testosterone; AA, athletic amenorrhea.
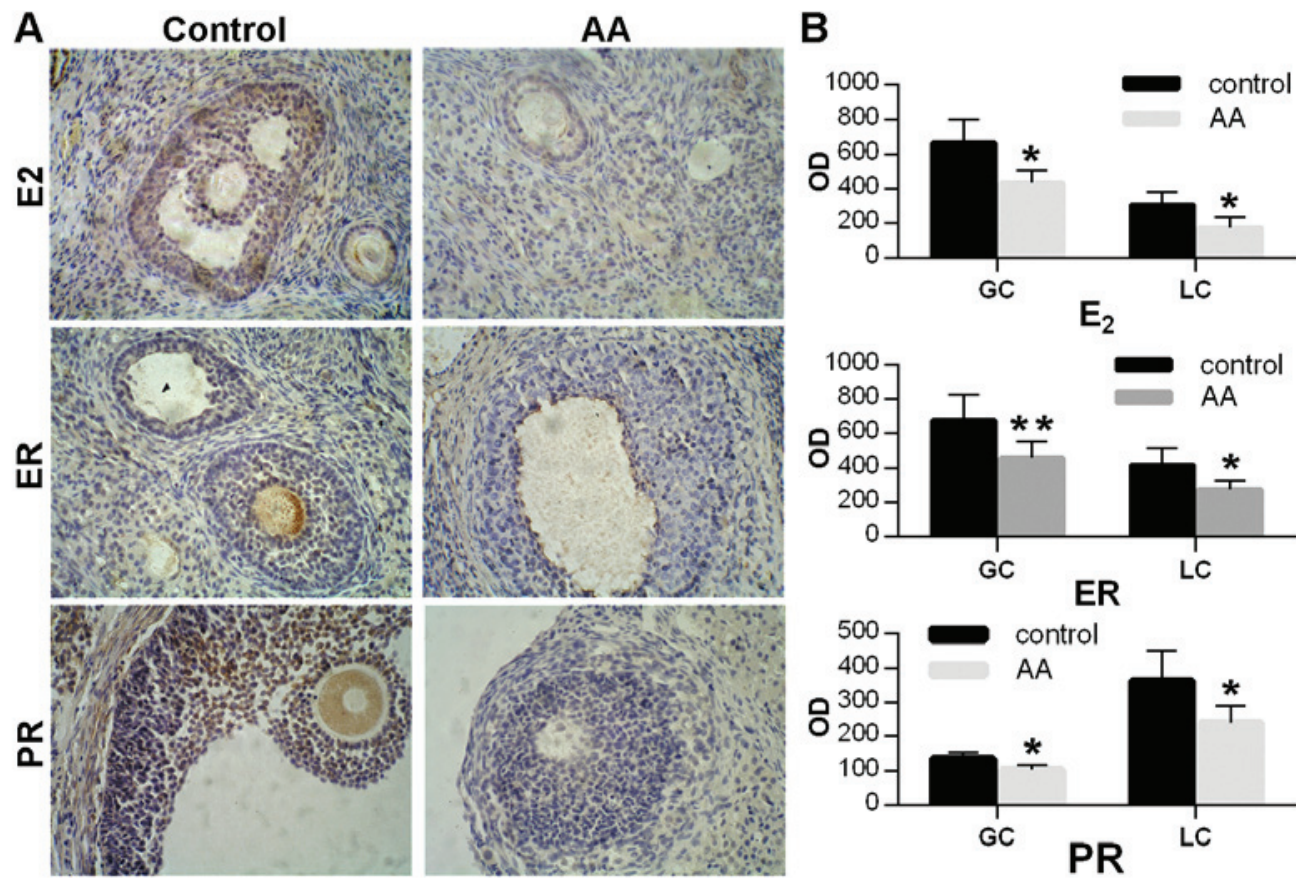

Figure 2. Expression levels of $\mathrm{E}_{2}, \mathrm{ER}$ and PR in GCs and LCs. (A) Immunohistochemical analysis for $\mathrm{E}_{2}, \mathrm{ER}$ and PR on ovarian tissue sections. (B) Expression levels of $\mathrm{E}_{2}$, ER and $\mathrm{PR}$ in the two cell types, expressed according to the OD values. $\mathrm{n}=20$ in each group. ${ }^{*} \mathrm{P}<0.05$ and ${ }^{* * *} \mathrm{P}<0.01$ vs. control group. GC, granular cell; LC, luteal cell; $\mathrm{E}_{2}$, estradiol; ER, estrogen receptor; PR, progesterone receptor.

group, which indicated the energy metabolism level was higher in control group.

\section{Discussion}

The incidence of AA is increasing worldwide, and this condition is one of the leading causes of infertility in female athletes (9). However, the underlying mechanisms and effective treatments of AA remain unclear. In the present study, the results demonstrated a decrease in the mtDNA copy number and protein level of ND2 in the ovaries of AA rats. These results indicate that mitochondrial dysfunction may be associated with the occurrence of AA.

The mitochondrion is the main organelle that supplies energy to the cells. The mitochondrial number varies among cells and tissues, and is associated with multiple functions. Mitochondria contain their own DNA, and a mammalian cell contains 1,000-10,000 copies of mtDNA (10). The mtDNA content is frequently used as a marker of mitochondrial density, while it also reflects the oxidative or ATP-producing capacity of tissues (11). Alterations in the mtDNA, which are reflected by the change of its copy number and its mutation, have been reported to be involved in a series of diseases and in organ dysfunction (4). Several studies have reported that the alterations of mtDNA are involved in the maturity, aging and lesion formation in the ovary $(12,13)$. Mutations in mtDNA can cause the abnormally expression of oxidative phosphorylation complex and abnormal transfer RNAs, which may be associated with polycystic ovarian syndrome patients (12). A previous study in pigs revealed that the copy number of mtDNA in the ovaries increased gradually along with sexual maturity (5). Devine et al (14) also reported that $20 \%$ of patients consulting for infertility presented signs of premature ovarian aging. In addition, ovarian aging is associated with reduced oocyte mtDNA content and mitochondrial dysfunction (13). Numerous studies have reported that the mtDNA content in the oocytes of aged women or of women with a diminished ovarian reserve is significantly lower when compared with that of young patients or those with a normal ovarian reserve (6-8). In the present study, the decreased copy number of mtDNA in the AA rats coincided with the dysfunction of the ovaries. The aforementioned results indicated that the decrease of the mtDNA copy number may suppress the oxidative phosphorylation process in ovarian cells and induce ovarian dysfunction, 
A
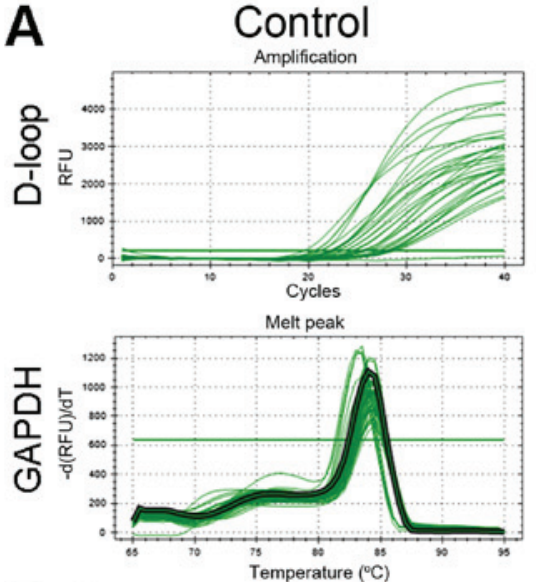

B

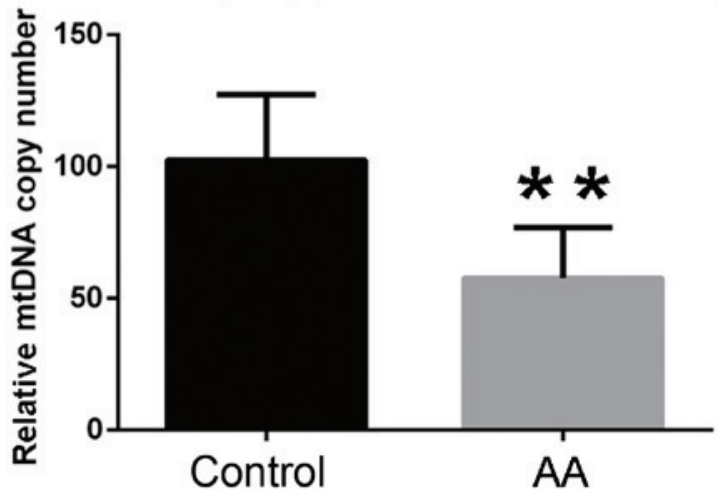

AA
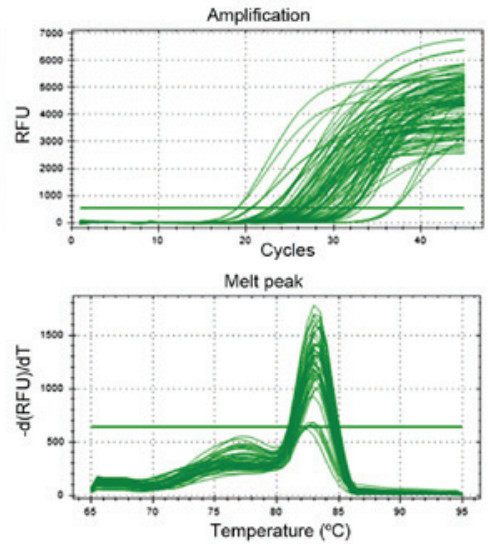
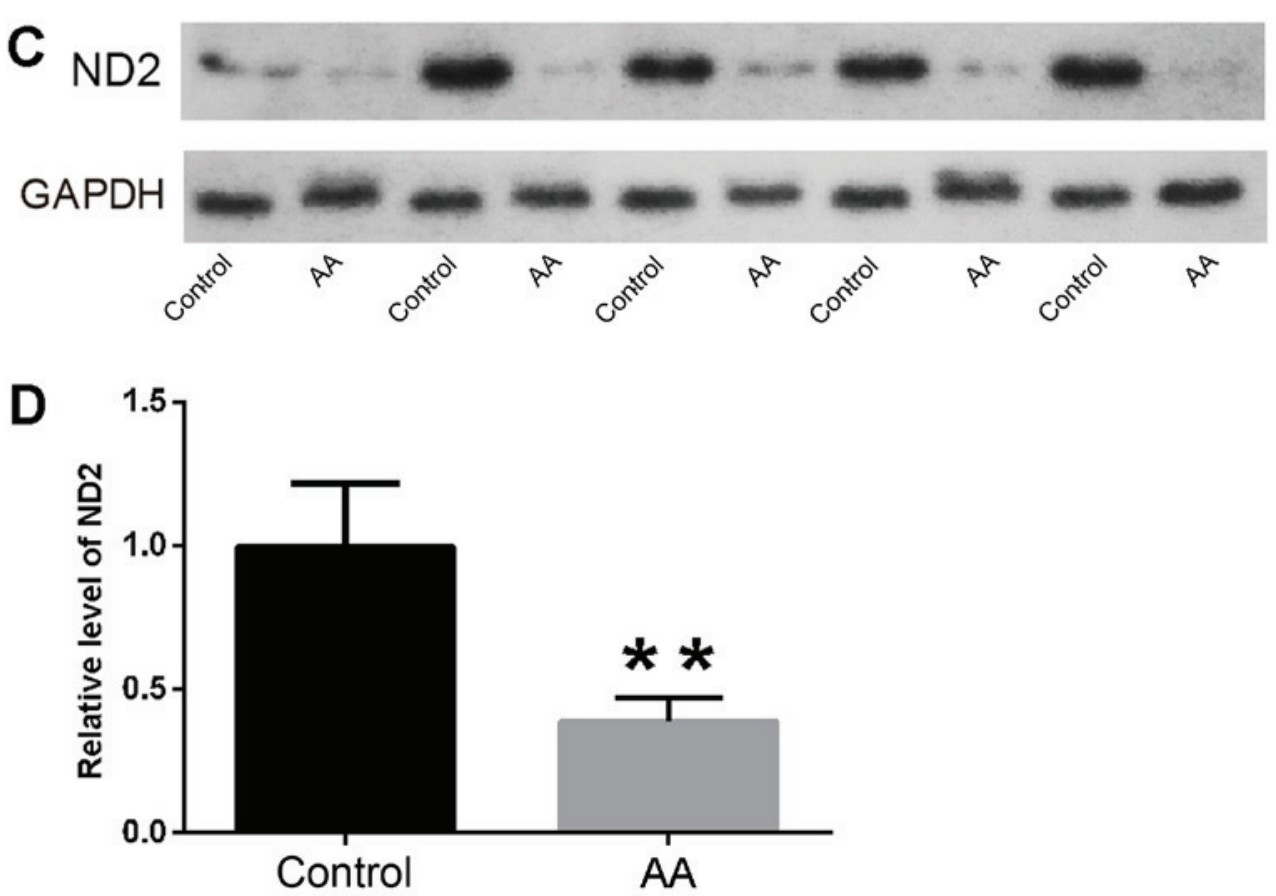

Figure 3. Decreased relative mtDNA copy number and ND2 expression in the ovarian tissues of AA rats. (A) Quantitative polymerase chain reaction amplification curves, and (B) relative mtDNA copy number in the control and AA groups. (C) Western blots of ND2 protein, and (D) quantified results of the relative protein level of ND2 in the ovaries. $\mathrm{n}=20$ in each group. ${ }^{* *} \mathrm{P}<0.01$ vs. control group. mtDNA, mitochondrial DNA; ND2, NADH dehydrogenase subunit 2 ; AA, athletic amenorrhea.

as well as the maturity of oocytes. However, further studies are required to clarify the underlying mechanisms.

NADH ubiquinone oxidoreductase (complex I) is the largest complex of the mitochondrial respiratory chain, and is composed by $\sim 45$ subunits. Of these, 7 subunits are directly encoded by the mitochondrial genome, including ND1-ND6 and ND4L (15). ND2 is one of the subunits of NADH that is a rate-limiting enzyme involved in oxidative phosphorylation (16). In the present study, it was observed that the protein level of ND2 was significantly decreased in AA rats, as determined by western blot analysis. In addition, the normal menstrual cycle and ovulation are controlled directly by the 
hormones that are secreted from the ovaries, including $E_{2}$, ER, PR, P, FSH, LH and T (17). The data of the present study revealed that these hormones in the ovaries or serum were markedly downregulated, which indicated the dysfunction of ovaries in the AA group. Accordingly, it is suggested that the decrease in the mtDNA copy number may induce the downregulation of ND2 expression and then suppress the oxidative phosphorylation, thus resulting in a lack of energy supply to the ovary and consequent ovarian dysfunction.

The morphological alterations of ovaries in the AA group observed by TEM confirmed that the mitochondria were the most affected organelles. The current results also indicated that mitochondrial dysfunction, particularly decreased mtDNA copy number and ND2 expression, may be considered as an early event during the development and/or progression of AA. Combined with the atrophy of ovaries in AA group, it is speculated that the atrophy may be associated with the mitochondrial dysfunction in female athletes.

In conclusion, the findings of the present study investigating AA rats suggest that excessive exercise may lead to morphological variations in the ovary, particularly to mitochondria. Furthermore, these morphological variations were accompanied with mitochondrial function disorder through decreased mtDNA content and ND2 expression. Thus, decrease in the mtDNA content and ND2 expression may be considered as the possible underlying mechanism of AA.

\section{Acknowledgements}

The authors would like to thank Mr. Xiao-Tao Zhou, Mr. Liu-Qi Wu and Miss Cong-Cong Zhang from the Chengdu Sport Institute (Chengdu, China), and Associate Professor Yan Fu from Southwest University for Nationalities (Chengdu, China) for their technical assistance.

\section{References}

1. Warren MP and Perlroth NE: The effects of intense exercise on the female reproductive system. J Endocrinol 170: 3-11, 2001.

2. Berz K, McCambridge T: Amenorrhea in the female athlete: What to do and when to worry. Pediatr Ann 45: e97-e102, 2016.

3. Stafford DE: Altered hypothalamic-pituitary-ovarian axis function in young female athletes: implications and recommendations for management. Treat Endocrinol 4: 147-154, 2005.
4. Picard M, Wallace DC and Burelle Y: The rise of mitochondria in medicine. Mitochondrion 30: 105-116, 2016.

5. Xie YM, Jin L, Chen XJ, He MN, Wang Y, Liu R, Li MZ and Li XW: Quantitative changes in mitochondrial DNA copy number in various tissues of pigs during growth. Genet Mol Res 14: 1662-1670, 2015.

6. Murakoshi Y, Sueoka K, Takahashi K, Sato S, Sakurai T, Tajima $\mathrm{H}$ and Yoshimura Y: Embryo developmental capability and pregnancy outcome are related to the mitochondrial DNA copy number and ooplasmic volume. J Assist Reprod Genet 30: 1367-1375, 2013.

7. Duran HE, Simsek-Duran F, Oehninger SC, Jones HW Jr and Castora FJ: The association of reproductive senescence with mitochondrial quantity, function and DNA integrity in human oocytes at different stages of maturation. Fertil Steril 96: 384-388, 2011

8. May-Panloup P, Chrétien MF, Jacques C, Vasseur C, Malthièry Y and Reynier P: Low oocyte mitochondrial DNA content in ovarian insufficiency. Hum Reprod 20: 593-597, 2005.

9. Dadgostar H, Razi M, Aleyasin A, Alenabi T and Dahaghin S: The relation between athletic sports and prevalence of amenorrhea and oligomenorrhea in Iranian female athletes. Sports Med Arthrosc Rehabil Ther Technol 1: 16, 2009.

10. Falkenberg M, Larsson NG and Gustafsson CM: DNA replication and transcription in mammalian mitochondria. Annu Rev Biochem 76: 679-699, 2007.

11. Hoeks J and Schrauwen P: Muscle mitochondria and insulin resistance: A human perspective. Trends Endocrinol Metab 23: 444-450, 2012

12. Zhuo G, Ding Y, Feng G, Yu L and Jiang Y: Analysis of mitochondrial DNA sequence variants in patients with polycystic ovary syndrome. Arch Gynecol Obstet 286: 653-659, 2012.

13. May-Panloup P, Boucret L, Chao de la Barca JM, Desquiret-Dumas V, Ferré-L'Hotellier V, Morinière C, Descamps P, Procaccio V and Reynier P: Ovarian ageing: The role of mitochondria in oocytes and follicles. Hum Reprod Update 22: 725-743, 2016.

14. Devine K, Mumford SL, Wu M, DeCherney AH, Hill MJ and Propst A. Diminished ovarian reserve in the United States assisted reproductive technology population: Diagnostic trends among 181,536 cycles from the society for assisted reproductive technology clinic outcomes reporting system. Fertil Steril 104: 612-619 e613, 2015.

15. Efremov RG, Baradaran R and Sazanov LA: The architecture of respiratory complex I. Nature 465: 441-445, 2010.

16. Wirth C, Brandt U, Hunte C and Zickermann V: Structure and function of mitochondrial complex I. Biochim Biophys Acta 1857: 902-914, 2016.

17. Reed BG and Carr BR: The Normal Menstrual Cycle and the Control of Ovulation. MDText.com, Inc., South Dartmouth MA, 2000. 